\title{
Improving schedule adherence based on dynamic signal control and speed guidance in connected bus system
}

\author{
Mingiie Hao and Yiming Bie \\ School of Transportation, Jilin University, Changchun, China \\ Le Zhang \\ School of Economics and Management, Nanjing University of Science and Technology, Nanjing, China, and \\ Chengyuan Mao \\ College of Engineering, Zhejiang Normal University, Jinhua, China
}

\begin{abstract}
Purpose - The purpose of this paper is to develop a dynamic control method to improve bus schedule adherence under connected bus system.

Design/methodology/approach - The authors developed a dynamic programming model that optimally schedules the bus operating speed at road sections and multiple signal timing plans at intersections to improve bus schedule adherence. First, the bus route was partitioned into three types of sections: stop, road and intersection. Then, transit agencies can control buses in real time based on all collected information; i.e. control bus operating speed on road sections and adjust the signal timing plans through signal controllers to improve the schedule adherence in connected bus environment. Finally, bus punctuality at the downstream stop and the saturation degree deviations of intersections were selected as the evaluation criteria in optimizing signal control plans and bus speeds jointly.

Findings - An illustrative case study by using a bus rapid transit line in Jinan city was performed to verify the proposed model. It revealed that based on the proposed strategy, the objective value could be reduced by $73.7 \%$, which indicated that the punctuality was highly improved but not to incur excessive congestion for other vehicular traffic.

Originality/value - In this paper, the authors applied speed guidance and the adjustment of the signal control plans for multiple cycles in advance to improve the scheduled stability; furthermore, the proposed control strategy can reduce the effect on private traffics to the utmost extend.
\end{abstract}

Keywords Dynamic programming, Speed guidance, Connected bus, Schedule adherence, Signal control

Paper type Research paper

\section{Introduction}

\subsection{Background}

In recent years, giving priority to public transportation has been deemed as an effective way to alleviate traffic congestion and reduce greenhouse gas emissions in most metropolis all around the world (Wang and Qu, 2015; Bie et al., 2015). Whether the passengers will choose to take buses or not depends on various factors, such as the fares, comfortability, walking distance, punctuality and so on. Specially, the punctuality is always regarded as an important indicator in evaluating the public transportation service quality and has attracted considerable attentions from both transit agencies and bus users consequently. However, it is extremely hard to guarantee that the bus could arrive at each station on time, because the operation of public transportation will be disturbed by numerous random factors, including the delay at intersections and the fluctuations according to passenger demand. If a bus is late, the bus will encounter more passengers along the

The current issue and full text archive of this journal is available on Emerald Insight at: https://www.emerald.com/insight/2399-9802.htm

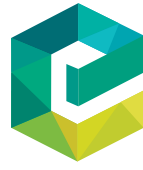

Journal of Intelligent and Connected Vehicles $3 / 2$ (2020) 79-88

Emerald Publishing Limited [ISSN 2399-9802] [DOI 10.1108/JICV-06-2020-0005] way, and these extra passengers will further delay the bus in turn. After enough time passes, buses bunch up and become irregular even if they departure with perfectly even headway, which aggravates the instability of transportation system seriously and lowers attention from passengers significantly. In this way, improving bus schedule adherence is highly important.

\subsection{Literature review}

To alleviate this problem, literature of dynamic control in public transportation has flourished in recent decades. Problems addressed by previous studies can be categorized into

(C) Mingjie Hao, Yiming Bie, Le Zhang and Chengyuan Mao. Published in Fournal of Intelligent and Connected Vehicles. Published by Emerald Publishing Limited. This article is published under the Creative Commons Attribution (CC BY 4.0) licence. Anyone may reproduce, distribute, translate and create derivative works of this article (for both commercial and non-commercial purposes), subject to full attribution to the original publication and authors. The full terms of this licence may be seen at http://creativecommons.org/licences/by/4.0/legalcode

This study is supported by the National Natural Science Foundation of China (No. 71771062), Natural Science Foundation of Zhejiang Province (LY18G030021) and China Postdoctoral Science Foundation (NO. 2019M661214).

Received 12 June 2020

Revised 16 July 2020

Accepted 22 July 2020 
two classes: scheduled-based method (Steiner and Irnich, 2018; Kumar et al., 2019; Chen and Sun, 2019) and headwaybased method (Zhang et al., 2020; Gkiotsalitis and Cats, 2018; Zhang and Lo, 2018). In the former group, the control is implemented according to the bus schedules, aiming at improving bus schedule adherence at each station, which is appropriate for low frequency transit lines, whereas headwaybased strategy is designed to keep headway close to the ideal along the route, and this method can be applied to high frequency transit lines.

However, all these methods largely rely on the bus holding strategies at predefined control points of bus lines. Chung et al. (2020) developed a connection protection (CP) model about holding control to protect the scheduled connection against delay of a trip. They aimed to minimize the expected total cost of all passengers related to the CP control, including the transfer passenger waiting time, the in-vehicle passenger waiting time and the downstream passenger waiting time. Liang et al. (2016) proposed the self-adaptive headways method with bus holding strategy considering both rear and front headways. In addition, compared with other models, the strategy could reduce unnecessary slack time. Dai et al. (2019) developed a predictive headwaybased bus holding strategy to address the bus-bunching problem.

Besides bus holding strategies, transit signal priority (TSP) is also one of the most promising and effective options to improve the reliability of transit systems. Gao et al. (2020) proposed a method to improve the reliability of bus services by signal priority control and speed guidance, considering the size of the upstream platoon and stop capacity constraint. Estrada et al. (2016) developed a new dynamic bus control strategy based on real-time bus tracking data at stops. In the strategy, extension of the green phase of traffic lights at intersections was used when a bus was significantly delayed. However, the sequence of each signal phase is fixed, and, consequently, the range of priority given to the bus is limited. In this way, it is hard to guarantee the schedule adherence precisely, especially when the bus's motion is far away from the predefined schedule because of some random disruptions. What is more, TSP will also bring huge impact to other vehicles, incurring excessive congestions.

In light of the above, there exists two critical issues in the literature. One common flaw is that at the curbside bus stops, bus holding will impede the operation of other transit vehicles, resulting in the traffic bottleneck. In this way, bus holding strategies are no longer applicable. Another limitation is that the literatures considering signal priority only adjust the timing plan of one signal cycle for the arrival bus. However, multiple road sections and intersections always exist among consecutive bus stops and the interaction between these multiple sections has usually been overlooked. The signal control could make up the delay because of uncontrolled randomness, which will further affect the velocity of bus in the next road section in turn. So we need to optimize the operation state of buses at each section jointly, generating the optimal control strategy to improve the bus schedule adherence.

\subsection{Contributions and rundown of the paper}

Recently, the connected vehicle technology has developed rapidly. With the help of this technic, the transit agencies could connect the real-time communications with buses and get traffic information timely, which makes it possible to control buses more precisely. The transit agencies could obtain bus operating data based on global positioning system equipment in real time and require buses to slow down at road section or extend the bus delay at intersection by signal adjustment (through signal controller) if buses arrive earlier compared with the previous scheduled plan, whereas speeding up or shortening bus delay at intersection could be applied to save the operation time. In such condition, there is no direct communication between the signal controller and buses. It is not necessary to place new detectors for buses on the roadside.

This paper aims at developing a new control strategy to avoid the irregularity of bus service. The major contributions of our paper are twofold. First, we formulate a dynamic programming model to optimize the bus speed at road section and signal timing plan at intersection jointly to improve bus schedule adherence. In our model, signal control means the adjustment of signal timing plan in multiple cycles. Thus, bus delay at intersections can be controlled by adjusting signal timing parameters of multiple cycles at intersections, which can avoid incurring significant congestion for general traffic. Compared with bus holding strategies, this method is more general as it is applicable to both bus bays and curbside bus stops. Second, the influence of upstream control on downstream travel time and punctuality is taken into consideration, and the control strategies for multiple sections are optimized coordinately. This optimization problem can be regarded as a multi-stage decision process and solved by dynamic programming approach.

The rest of this paper is organized as follows. Section 2 presents the dynamic programming model and methodology; a case study is presented in Section 3. Section 4 makes some discussions; Section 5 concludes the paper.

\section{Methodology}

As shown in Figure 1, the bus route is divided into three types of sections: stop, road and intersection. The precise bus control proposed by this paper is on the premise of this division. Next, we will define the three sections one by one. The stop section is defined as the region from the bus decelerating and entering the station to accelerating and departing from it. In light traffic condition, the bus can operate in larger speed, and, consequently, it will take more time and longer distance for buses to decelerate from the present speed to 0 and accelerate from 0 to normal operation state. When the speed is up to the maximum, the corresponding distance of stop region will also reach to the highest. In addition, we assume that there is no control strategy applied in the stop section. Thus, the distance of stop section can be set as constant, equal to the sum of

\section{Figure 1 Three types of sections on one bus route}

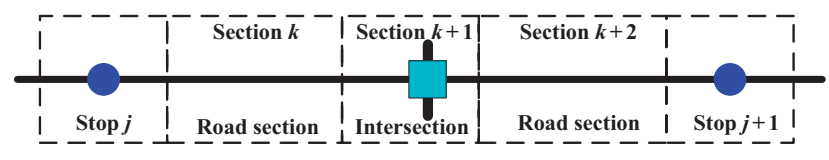


platform length, maximum deceleration distance and maximum acceleration distance. The length of intersection is set as the maximum queue length incurred by the red phase of traffic signals. Other sectors along the bus route are road sections.

The operation of buses is disturbed by various random factors, i.e. traffic demand, passenger needs, the vagaries of bus drivers, where passenger arrival rate accounts for a great proportion. The number of waiting passengers determines bus dwell time, and these errors can only be eliminated by other control methods. A new dynamic programming model is described in this section, combing the speed guidance and signal control along the whole bus route and avoiding the inapplicability to curbside bus stops caused by conventional control strategies.

\subsection{Problem description}

As shown in Figure 1, from stop $j$ to $j+1$, the bus will pass through section $k, k+1$ and $k+2$ successively. The punctuality at stop $j+1$ is determined by the travel time at each section, while the three sections also interact mutually. So we need to optimize the control scheme at each section jointly.

We divide the control scheme from stop $j$ to $j+1$ into multiple stages, according to the partition of bus lines discussed before. Each stage starts from the entering of buses into the corresponding route section. Then this optimization problem is a typical multistage decision process and can be solved by dynamic programming as shown in Figure 2. In the figure, $t_{k}$, $t_{k+1}$ and $t_{k+2}$ are entering time of buses into section $k, k+1$ and $k+2$ respectively. $t_{j+1}$ is the arrival time of buses at stop $j+1$. A brief review of known, useful facts now follows.

As for the control process, the central controllers need to optimize the control schedule at the beginning of each stage through the following time period to $t_{j+1}$ step by step based on the traffic information collected in real time. Then the optimal plan will be executed for the corresponding stage.

\subsection{Model development}

In the model, we assume that transit agency could get the exact bus location and signal timing plans of intersections in real time in V2I environment. The objective of our analysis is to keep the buses along a given route operating with the predefined regular schedule while avoiding the side effect on other vehicular traffic caused by the proposed control scheme. We shall define the set of state variables at stage $k$ as $x_{k}=\left\{t_{k}\right\}$, where $t_{k}$ is moment of buses entering into the section $k$ and the size of set $x_{k}$ is defined as $E_{k}$ and $x_{k}\left(e_{k}\right)$ indicates the $e_{k}-t h$ element of set $x_{k}\left(1 \leq e_{k} \leq E_{k}\right)$. At stage $k$, the decision variable is set as $u_{k}$, the average speed of buses in road section $k$. In V2I environment, travel speed of large vehicles on the road section can be evaluated in real time. The minimum and maximum values of these speeds can be set as $v_{k}^{\min }$ and $v_{k}^{\max }$, which are also the boundaries of bus operation speed. Before applying the dynamic algorithm, we discretize the operation speed $u_{k}$ into $D_{k}$ points; i.e. $u_{k} \in\left\{v_{k}^{\text {min }}, v_{k}^{\text {min }}+\frac{v_{k}^{\text {max }}-v_{k}^{\text {min }}}{D_{k}-1}, v_{k}^{\text {min }}+2 \times \frac{v_{k}^{\text {max }}-v_{k}^{\text {min }}}{D_{k}-1}, \cdots, v_{k}^{\text {max }}\right\}$. Thus $u_{k}(1)=v_{k}^{\min }$ and $u_{k}\left(D_{k}\right)=v_{k}^{\max }$. Here we discretize the bus speed into $D_{k}$ points and as $D_{k}$ increases, the speed guidance will be more precise. Almost the same notions and decision variables apply for road section $k+2$ except the stage index. At intersection $k+1$, each decision is associated with the staring time of signal phases. Different from previous studies only considering all the phases in one signal cycle, we focus on the extension or contraction of all the signal cycles from the bus entering the road section $k$ to leaving intersection $k+1$, which can enlarge the adjustment range of signal plan and lower the influence on other transit vehicles to the maximum extent.

In our model, $v_{k}^{\min }$ and $v_{k}^{\max }$ are two vital parameters and their values will affect the final optimal control significantly. Theoretically, $v_{k}^{\min }$ could take value as $0 \mathrm{~km} / \mathrm{h}$. However, it will cause the severe negative feeling among passengers when the buses are operated approximately to $0 \mathrm{~km} / \mathrm{h}$ on the road section. Thus, the value of $v_{k}^{\min }$ is taken according to the investigation among passengers, lowering their discomfort to a certain degree (e.g. $10 \mathrm{~km} / \mathrm{h}$ ). $v_{k}^{\max }$ is influenced by real-time traffic conditions; i.e. under low-flow condition, $v_{k}^{\max }$ can be larger (e.g. $50 \mathrm{~km} / \mathrm{h}$ ) compared with that in high flow (e.g. $25 \mathrm{~km} / \mathrm{h}$ ). In V2I environment, the 85 th-percentile operating speed of all buses on the same road section can be acquired in real time and used to determine $v_{k}^{\max }$.

To optimize the bus states of different sections jointly, we build the state transfer functions as follows:road section $k \rightarrow$ intersection $k+1$ :

$$
x_{k+1}=t_{k}+L_{k} / v_{k}
$$

intersection $k+1 \rightarrow$ road section $k+2$ :

$$
x_{k+2}=x_{k+1}+T_{k+1}\left(u_{k+1}\right)=x_{k+1}+f\left(u_{k+1}\right)
$$

Figure 2 Illustration of the multiple-stage decision process

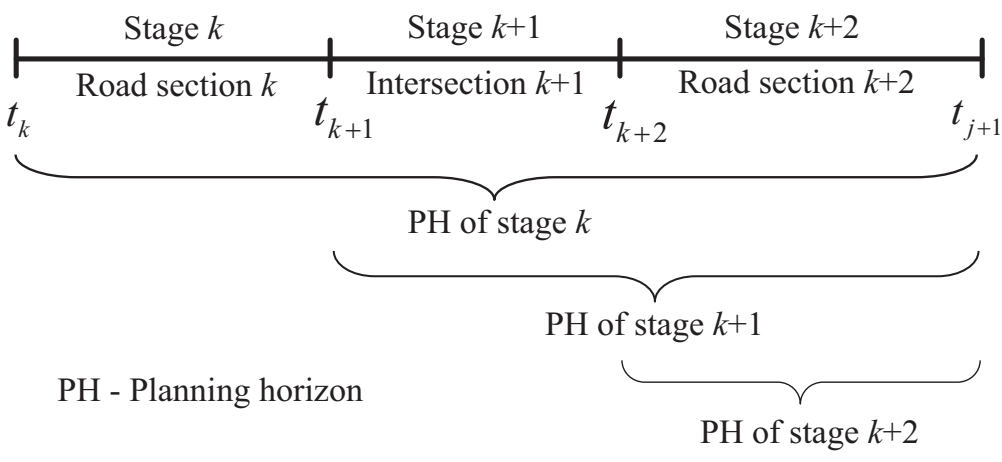


Mingjie Hao, Yiming Bie, Le Zhang and Chengyuan Mao

$$
x_{j+1}=x_{k+2}+L_{k+2} / v_{k+2}
$$

where $L_{k}$ is the length of section $k, T_{k+1}$ is the travel time of buses across section $k+1$, which is a function of signal control plan denoted as $f\left(u_{k+1}\right)$. Next, we will explain these state transfer functions in details and model the one-step cost functions stage by stage.

\section{Stage $k$ :}

We assume that the bus enters section $k$ at $t_{k+1}$ and the control also begins to be implemented at the same time. Thus, state variable set $x_{k}$ includes only one element, $t_{k}$ i.e.:

$$
\begin{gathered}
E_{k}=1 \\
u_{k}=\left\{v_{k}^{\text {min }}, v_{k}^{\text {min }}+\frac{v_{k}^{\text {max }}-v_{k}^{\text {min }}}{D_{k}-1}, v_{k}^{\text {min }}+2 \times \frac{v_{k}^{\text {max }}-v_{k}^{\text {min }}}{D_{k}-1}, \cdots, v_{k}^{\text {max }}\right\}
\end{gathered}
$$

As the speed at stage $k$ will affect the signal control and speed guidance at the following stages, thus the one-step cost function at this stage is set as constant, i.e.:

$U_{k}\left(t_{k}, 1\right)=U_{k}\left(t_{k}, 2\right)=\cdots=U_{k}\left(t_{k}, d_{k}\right)=\cdots=U_{k}\left(t_{k}, D_{k}\right)=\rho$

$U_{k}\left(t_{k}, d_{k}\right)$ is the utilization cost at state $t_{k}$ when the bus is operated with speed $u_{k}\left(d_{k}\right)\left(1 \leq d_{k} \leq D_{k}\right)$.

Stage $k+1$ :

The traffic signal consists of $M$ phases and buses are controlled by phase 1 . We assume that each signal cycle begins with the green starting time of phase 1 . We shall define $t_{i}^{m, s}$ and $t_{i}^{m, e}$ as the starting and ending time of phase $m$ in signal cycle $i$, respectively. Thus the starting time $C_{i}^{s}$ and ending time $C_{i}^{e}$ of signal cycle $i$ would simply be:

$$
\begin{gathered}
C_{i}^{s}=t_{i}^{1, s} \\
C_{i}^{e}=t_{i}^{M, e} \\
C_{i+1}^{s}=C_{i}^{e}=t_{i+1}^{1, s} \\
t_{i}^{m, s} \leq t_{k}<t_{i}^{m, e}
\end{gathered}
$$

where equation (1) specifies that the bus runs into section $k$ during the phase $m$ cycle $i$. If the bus passes through the intersection during the $n t h$ cycle, then total $(n-i+1)$ signal cycles could be controlled, while only phases $m$ to $M$ are available in the $i t h$ cycle. As in this paper, we assume that there is only one bus between stops $j$ and $j+1$. Obviously, the ending time of one phase is equal to the starting time of the following phase as shown in equation (9), so we only need to optimize the staring time of each phase from cycle $i$ to $n$ satisfying the following constraints:
Volume $3 \cdot$ Number $2 \cdot 2020 \cdot 79-88$

$$
\left\{\begin{array}{c}
\left(1-\lambda_{1}\right) \tilde{g}_{i}^{j} \leq g_{i}^{j} \leq\left(1+\lambda_{2}\right) \tilde{g}_{i}^{j} \quad m \leq j \leq M \\
\left(1-\lambda_{1}\right) \tilde{g}_{n^{\prime}}^{j} \leq g_{n^{\prime}}^{j} \leq\left(1+\lambda_{2}\right) \tilde{g}_{n^{\prime}}^{j} \quad 1 \leq j \leq M, \quad i<n^{\prime} \leq n \\
t_{n^{\prime}}^{j, s}=t_{n^{\prime}}^{1, s}+\sum_{m=1}^{j-1} g_{n^{\prime}}^{m} i<n^{\prime} \leq n \\
t_{n^{\prime}}^{j, e}=t_{n^{\prime}}^{j, s}+g_{n^{\prime}}^{j} \quad 1 \leq j \leq M, \quad i<n^{\prime} \leq n \\
\lambda_{1} \geq 0, \lambda_{2} \geq 0 \\
\tilde{g}_{i}^{j}=\frac{q_{j}\left(\tilde{C}_{i}-A\right)}{q}
\end{array}\right.
$$

where $\tilde{g}_{i}^{j}$ is the allocated green time of phase $j$ cycle $i$ without considering bus control, which can be calculated by equation (12); $g_{i}^{j}$ is the allocated green time of phase $j$ cycle $i$ after applying the bus control; $\lambda_{1}$ and $\lambda_{2}$ specify the variation range of green time; $q_{j}$ is the traffic flow of phase $j ; q$ is the total traffic volume of all the critical lanes; $\tilde{C}_{i}$ is the signal cycle length without considering bus control; and $A$ is the total sum of intergreen time of $M$ phases.

For a given state variable $x_{k+1}\left(e_{k+1}\right) \in\left[x_{k+1}(1), x_{k+1}\left(E_{k+1}\right)\right]$, we would predict $\vec{x}_{k+1}\left(e_{k+1}\right)$, the time that the bus joins in the queue behind the stop line as follows:

$$
L_{b}(t)=L_{k+1}-v_{k+1}\left[t-x_{k+1}\left(e_{k+1}\right)\right] \quad t \geq x_{k+1}\left(e_{k+1}\right)
$$

$$
\begin{aligned}
L_{k+1}= & Q_{i-1}^{1}+\max \{0,(n-i+2) \\
& {\left.\left[q_{1}-\frac{S_{1}\left(1-\lambda_{1}\right) g_{i}^{1}}{\left(1-\lambda_{1}\right) g_{i}^{1}+\sum_{m=2}^{M}\left(1-\lambda_{2}\right) g_{i}^{m}}\right]\right\} }
\end{aligned}
$$

$L_{k+1}^{n}(t)=\left\{\begin{array}{c}L_{k+1}^{n-1}\left(t_{n-1}^{1, e}\right)+\left(t-t_{n-1}^{1, e}\right) v_{k+1}^{f} \varepsilon_{k+1}-v_{k+1}^{f}\left(t-t_{n}^{1, s}\right) \quad t_{n}^{1, s} \leq t \leq t_{n}^{1, e} \\ L_{k+1}^{n}\left(t_{n-1}^{1, e}\right)+\left(t-t_{n}^{1, e}\right) v_{k+1}^{f} \varepsilon_{k+1} \quad t_{n}^{1, e} \leq t \leq t_{n}^{M, e}\end{array}\right.$

where in equation (13) $x_{k+1}\left(e_{k+1}\right) \in\left[x_{k+1}(1), x_{k+1}\left(E_{k+1}\right)\right] L_{b}(t)$ is the distance between the stop line and the current location of the bus at time $t ; e_{k+1}$ is index of state variable $x_{k+1} ; v_{k+1}$ is the predefined speed at section $k+1$. equation (14) indicates the length of section $k+1$, where $Q_{i-1}^{1}$ is the uncontrolled queue length at the end of Phase 1 cycle $i-1 . S_{1}$ is the saturation flow for the critical lane of phase 1 . The second term of equation (14) indicates the possible queue length incurred by dynamic bus control method. The queue length of section $k+1$ at time $t$ cycle $n$ is given by equation (15), where $v_{k+1}^{f}$ is the free flow speed at section $k+1 ; \varepsilon_{k+1}$ is the ratio of road traffic density to jam density.

Then we combine equations (13) and (15) together. If $L_{b}(t)=L_{k+1}^{n}(t)$, then the bus joins in the queue at phase 1 cycle $n$ and $t=\vec{x}_{k+1}\left(e_{k+1}\right)$. Thus, the distance between the current location of bus and stop line is $L_{k+1}^{n}\left(\vec{x}_{k+1}\left(e_{k+1}\right)\right)$. Then, we consider the following constraints: 


$$
\begin{gathered}
g_{b u s}^{1}=L_{k+1}^{n}\left(\vec{x}_{k+1}\left(e_{k+1}\right)\right) / v_{k+1}^{f} \\
t_{n}^{1, s} \leq \vec{x}_{k+1}\left(e_{k+1}\right)<t_{n}^{1, e} \\
{\left[t_{n}^{1, e}-\vec{x}_{k+1}\left(e_{k+1}\right)\right] \geq g_{b u s}^{1}}
\end{gathered}
$$

where $g_{b u s}^{1}$ denotes the time for the staring wave transferred to the bus and constraints (18) specifies that the residual green time should be no less than $g_{b u s}^{1}$. Obviously, the bus will pass through the intersection at the cycle $n$ if both constraints (17) and (18) are satisfied. Otherwise, the bus will wait until the next cycle $n+1$. Then the entering time into section $k+2$ can be derived in two scenarios as follows:

Case 1: $t_{n}^{1, s} \leq \vec{x}_{k+1}\left(e_{k+1}\right)<t_{n}^{1, e} \&\left[t_{n}^{1, e}-\vec{x}_{k+1}\left(e_{k+1}\right)\right] \geq g_{b u s}^{1}$ Bus gets to the stop line at phase 1 cycle $n$, then delay $\eta$ at intersection is 0 :

$$
\eta=0
$$

and the entering time of bus into section $k+2$ is as follows:

$$
x_{k+2}\left[x_{k+1}\left(e_{k+1}\right)\right]=\vec{x}_{k+1}\left(e_{k+1}\right)+g_{b u s}^{1}
$$

Case 2:

The arriving time of bus at the stop line is during the red phase and the bus will wait until the following green time starts at the next light cycle $n+1$, or the residual green time is not enough for the bus to run across the intersection, then the added delay is chosen to be:

$$
\begin{gathered}
\eta=t_{n+1}^{1, s}-\vec{x}_{k+1}\left(e_{k+1}\right)+\eta_{1} \\
\eta_{1}=\left[B_{n}^{1}+\left(\vec{x}_{k+1}\left(e_{k+1}\right)-t_{n}^{1, e}\right) \cdot q_{1}\right] / S_{1} \\
B_{n}^{1}=q_{1} \sum_{i=1}^{M} g_{n}^{i}-S_{1} g_{n}^{1}
\end{gathered}
$$

where:

$\eta_{1}=$ delay because of discharging the queue before the bus;

$B_{n}^{i}=$ number of undischarged vehicles at phase $i$ cycle $n$; and

$S_{1}=$ saturation flow for the critical lane of phase 1 .

Then the bus will enter section $k+2$ at:

$$
x_{k+2}\left[x_{k+1}\left(e_{k+1}\right)\right]=\vec{x}_{k+1}\left(e_{k+1}\right)+\eta
$$

Then, in section $k+1$, the size of decision variables is of the following form:

$$
D_{k+1}= \begin{cases}\left(\lambda_{1}+\lambda_{2}\right) \prod_{j=m}^{M} \tilde{g}_{i}^{j} \cdot \prod_{j=m}^{M} \tilde{g}_{i+1}^{j} \cdots \prod_{j=m}^{M} \tilde{g}_{n}^{j} \quad \text { for case 1 } \\ \left(\lambda_{1}+\lambda_{2}\right) \prod_{j=m}^{M} \tilde{g}_{i}^{j} \cdot \prod_{j=m}^{M} \tilde{g}_{i+1}^{j} \cdots \prod_{j=m}^{M} \tilde{g}_{n+1}^{j} & \text { for case 2 }\end{cases}
$$

As the control will influence other vehicles, to model this effect in a simple way, we shall use saturation degree deviation to measure this effect; i.e.:
$\mathrm{U}_{k+1}\left(e_{k+1}, d_{k+1}\right)= \begin{cases}\frac{\left|\sum_{j=1}^{n} \delta_{j}-\sum_{j=1}^{n} \tilde{\delta}_{j}\right|}{\sum_{j=1}^{n} \tilde{\delta}_{j}} \cdot \omega & \text { for case 1 } \\ \frac{\left|\sum_{j=1}^{n+1} \delta_{j}-\sum_{j=1}^{n+1} \tilde{\delta}_{j}\right|}{\sum_{j=1}^{n+1} \tilde{\delta}_{j}} \cdot \omega \text { for case 2 }\end{cases}$

$$
\left\{\begin{array}{l}
\delta_{j}^{m}=\frac{B_{j}^{m}+q_{m} C_{j}}{g_{j}^{m} S_{j}} \\
\delta_{j}=\max \left(\delta_{j}^{1}, \delta_{j}^{2} \cdots \delta_{j}^{M}\right)
\end{array}\right.
$$

where $\tilde{\delta}_{j}$ is the saturation degree of signal cycle $j$ without considering the bus control; $\delta_{j}^{m}$ is the saturation degree of phase $m$ cycle $j ; q_{m}$ is the traffic flow of phase $m$; $S_{j}$ is the saturation flow for the critical lane of phase $j ; \omega$ is a weight factor. Equation (27) indicates the optimal utilization cost at state $x_{k+1}\left(e_{k+1}\right)$.

Saturation degree deviation of the intersection is used to denote the cost function of stage $k+1$. This is because in this stage the change of signal control plan will affect the operations of buses and private cars. However, the change of bus operation can be reflected at stage $k+2$. Thus, in this stage only the private cars are considered. We also want to explain why we use saturation degree deviation instead of other indexes, such as average vehicle delay, queue length or stop rate. This is because the calculation equation of saturation degree deviation is the simplest among these indexes. Besides, the increase of saturation degree will result in the increase of average vehicle delay, queue length and stop rate, and the decrease of capacity and thus saturation degree deviation is the most essential criteria compared with other criteria.

Stage $k+2$ :

At stage $k+2$, the decision variables are speed as shown in equation (28) and non-punctuality is chosen as the utilization cost of this stage to evaluate schedule adherence of the bus system as shown in equation (29):

$$
\begin{gathered}
u_{k+2}=\left\{v_{k+2}^{\min }, v_{k+2}^{\min }+\frac{v_{k+2}^{\max }-v_{k+2}^{\min }}{D_{k+2}-1}, v_{k+2}^{\min }\right. \\
\left.+2 \times \frac{v_{k+2}^{\max }-v_{k+2}^{\min }}{D_{k+2}-1}, \cdots, v_{k+2}^{\max }\right\} \\
U_{k+2}\left(e_{k+2}, d_{k+2}\right)=\frac{\left|t_{j+1}\left(e_{k+2}, d_{k+2}\right)-t_{j+1}^{s}\right|}{H}
\end{gathered}
$$

The schedule is defined by the term $t_{j+1}^{s}$, at which bus is expected to arrive at stop $j+1$, and $H$ is the service headway; $t_{j+1}\left(e_{k+2}, d_{k+2}\right)$ is the actual arrival time under state $x_{k+1}\left(e_{k+2}\right)$ and operating speed $u_{k+2}\left(d_{k+2}\right)\left(1 \leq d_{k+2} \leq D_{k+2}\right)$.

The optimal objective cost for the segment between stop $j$ and $j+1$ is defined as the sum of these three stages' utilization cost and denoted by $\mathcal{F}^{*}$. Backward recursion solution can be used to solve this typical dynamic model. Overall, a brief 
introduction of the backward recursion solution is displayed below:

- Step 1: At stage $k+2$, enumerate all the possible entering time into stage $k+2$; calculate the corresponding non-punctuality according to equation (29) when buses are operated with all the possible discrete speeds. For a given state $x_{k+2}\left(e_{k+2}\right)$ in stage $k+2$, the corresponding optimal utilization cost is $\mathcal{H}_{k+2}^{*}\left(e_{k+2}\right)=\min _{1 \leq d_{k+2} \leq D_{k+2}} U_{k+2}\left(e_{k+2}, d_{k+2}\right)$, where $1 \leq$ $e_{\mathrm{k}+2} \leq E_{k+2}$.

- Step 2: At stage $k+1$, calculate all the possible saturation degree deviations under different signal timing plans. Different signal timing planning will result in different saturation degree deviations. For any state variable $x_{k+1}\left(e_{k+1}\right)\left(1 \leq e_{k+1} \leq E_{k+1}\right)$, the optimal utilization cost is defined as $\mathcal{F}_{k+1}^{*}\left(e_{k+1}\right)=\min _{1 \leq d_{k+1} \leq D_{k+1}}\left(U_{k+1}\left(e_{k+1}, d_{k+1}\right)+\right.$ $\left.\mathcal{H}_{k+2}^{*}\left(e_{k+2}\right)\right)$, where $x_{k+2}\left(e_{k+2}\right)=x_{k+1}\left(e_{k+1}\right)+f$ $\left(u_{k+1}\left(d_{k+1}\right)\right)$ according to equation (2).

- Step 3: Similarly to stage $k$, consider all the possible speeds. After calculating the utilization cost at stage $k$, find the minimum total objective value $\mathcal{f}^{*}$, i.e., the sum of utilization cost at each stage. $\mathcal{H}^{*}=\min _{1 \leq d_{k} \leq D_{k}}\left(U_{k}\left(e_{k}, d_{k}\right)+\mathcal{F}_{k+1}^{*}\left(e_{k+1}\right)\right)$, where $x_{k+1}\left(e_{k+1}\right)=t_{k}+\frac{L_{k}}{u_{k}\left(d_{k}\right)}$. Because $U_{k}\left(e_{k}, d_{k}\right) \equiv \rho$ as described before, then the final optimal utilization cost can be simplified as $\mathcal{F}^{*}=\rho+\min _{1 \leq e_{k+1} \leq E_{k+1}} \mathcal{F}_{k+1}^{*}\left(e_{k+1}\right)$.

\section{Case study}

\subsection{Description of the bus route}

In this section, the proposed dynamic programming model is validated based on the Bus Rapid Transit (BRT) Line 13 (BRT 13) in Jinan city, China. The length of BRT 13 is $7.9 \mathrm{~km}$ including 14 stops and 10 signalized intersections, as shown in Figure 3. The location of each stop, dwell time and passenger flow are presented in Table 1 . The detailed information of each intersection, such as traffic flow and signal timing, is shown in Table 2. The departing time intervals in peak hour and off-peak hours are $6 \mathrm{~min}$ and $9 \mathrm{~min}$, respectively. In this study, the peak hour is taken as an example to conduct the case study.

In this section, two plans will be compared via the case study. Plan I is the current plan on BRT 13, and there is no special control method. In Plan II, the proposed dynamic bus control method is applied to BRT 13.

The whole bus route is divided into three types of sections: stop, road and intersection. The length of stop section is fixed, which is 50 meters. The bus route contains 23 road sections and the length of each road section is shown in Table 3 . The distances between two successive intersections are summarized in Table 4. The minimum and maximum allowed speed is $2.8 \mathrm{~m} / \mathrm{s}$ and $8.3 \mathrm{~m} / \mathrm{s}$. In addition, the weight factor $\omega$ is equal to $0.2, \lambda_{1}=\lambda_{2}=0.2$.

\subsection{Comparisons and evaluations}

\subsubsection{Punctuality of the transit system}

There are two different plans in the comparisons, which are Plan I and Plan II. Average non-punctuality rates at different stops under two plans are shown in Figure 4, and there are significant differences between Plan I and Plan II. Under Plan II, buses arrive at most stations punctually.

Bus operations are intrinsically unstable and once a bus arrives late at a stop, it will encounter more passengers at

Table 1 Bus operation data on BRT 13

\begin{tabular}{cccccc}
\hline $\begin{array}{c}\text { Stop } \\
\text { no. }\end{array}$ & $\begin{array}{c}\text { Stop } \\
\text { distance } \\
(\mathrm{m})\end{array}$ & $\begin{array}{c}\text { Boarding } \\
\text { volume } \\
(\text { pax })\end{array}$ & $\begin{array}{c}\text { Alighting } \\
\text { volume } \\
(\text { pax })\end{array}$ & $\begin{array}{c}\text { Dwell } \\
\text { time (s) }\end{array}$ & $\begin{array}{c}\text { Inner-stop } \\
\text { travel time } \\
(\mathbf{s})\end{array}$ \\
\hline $\mathbf{1}$ & - & 130 & 0 & 20 & - \\
$\mathbf{2}$ & 1500 & 140 & 0 & 31 & 233 \\
$\mathbf{3}$ & 889 & 110 & 0 & 21 & 160 \\
$\mathbf{4}$ & 586 & 120 & 70 & 29 & 109 \\
$\mathbf{5}$ & 346 & 150 & 60 & 34 & 83 \\
$\mathbf{6}$ & 434 & 130 & 60 & 33 & 102 \\
$\mathbf{7}$ & 445 & 140 & 80 & 36 & 104 \\
$\mathbf{8}$ & 460 & 120 & 40 & 29 & 108 \\
$\mathbf{9}$ & 311 & 60 & 160 & 27 & 85 \\
10 & 348 & 10 & 150 & 23 & 97 \\
11 & 374 & 0 & 130 & 27 & 80 \\
12 & 540 & 20 & 110 & 24 & 114 \\
13 & 997 & 0 & 120 & 24 & 163 \\
14 & 670 & 0 & 150 & 32 & 122 \\
\hline
\end{tabular}

Table 2 Data of intersections on BRT 13

\begin{tabular}{|c|c|c|c|}
\hline $\begin{array}{l}\text { Intersection } \\
\text { no. }\end{array}$ & $\begin{array}{c}\text { Distance to } \\
\text { next } \\
\text { intersection (m) }\end{array}$ & $\begin{array}{l}\text { Traffic flow of } \\
\text { phases (pcu/h) }\end{array}$ & Timing plan (s) \\
\hline 1 & - & {$\left[\begin{array}{lllll}750 & 141 & 366 & 159\end{array}\right]$} & {$\left[\begin{array}{lllll}128 & 56 & 17 & 24 & 19\end{array}\right]$} \\
\hline 2 & 1120 & [738 148413 148] & {$\left[\begin{array}{lllll}122 & 48 & 18 & 27 & 17\end{array}\right]$} \\
\hline 3 & 724 & [769 175 594] & [109 442135$]$ \\
\hline 4 & 530 & {$\left[\begin{array}{llll}868 & 169 & 638\end{array}\right]$} & [141 5926 47] \\
\hline 5 & 790 & [792 181453 136] & [159 583039 20] \\
\hline 6 & 470 & {$\left[\begin{array}{llll}741 & 185 & 582\end{array}\right]$} & {$\left[\begin{array}{lllll}136 & 55 & 29 & 43\end{array}\right]$} \\
\hline 7 & 450 & [842 164442 137] & [178 613443 28] \\
\hline 8 & 320 & [834 132 633] & {$\left[\begin{array}{lllll}108 & 47 & 16 & 36\end{array}\right]$} \\
\hline 9 & 384 & [806 161618 ] & {$\left[\begin{array}{llllll}134 & 56 & 24 & 45\end{array}\right]$} \\
\hline 10 & 310 & [710 158473 131] & [137 $\left.46 \begin{array}{llll}13 & 34 & 20\end{array}\right]$ \\
\hline
\end{tabular}

Figure 3 BRT 13 line

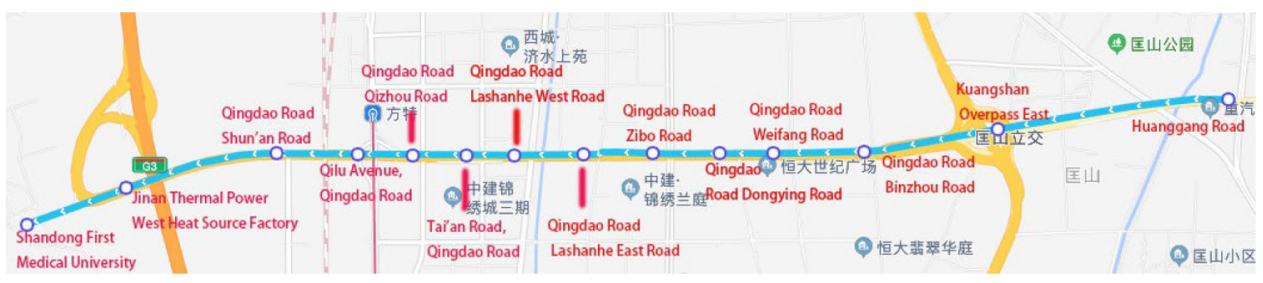


Table 3 Length of each road section on BRT 13

\begin{tabular}{|c|c|c|c|c|c|c|c|c|c|c|c|c|}
\hline Road section no. & 1 & 2 & 3 & 4 & 5 & 6 & 7 & 8 & 9 & 10 & 11 & $\overline{12}$ \\
\hline Length (m) & 370 & 700 & 37 & 675 & 26 & 416 & 89 & 21 & 253 & 86 & 278 & 70 \\
\hline Road section no. & 13 & 14 & 15 & 16 & 17 & 18 & 19 & 20 & 21 & 22 & 23 & \\
\hline Length (m) & 277 & 83 & 157 & 70 & 228 & 36 & 187 & 75 & 475 & 930 & 634 & \\
\hline
\end{tabular}

Table 4 Adjusted timing plans in different cycles for a bus

\begin{tabular}{|c|c|}
\hline Intersection no. & Timing plans in adjusted cycles \\
\hline 1 & None \\
\hline 2 & None \\
\hline 3 & [115 4623 37]; [ [115 4623 37]; [111 $\left.46 \begin{array}{lll}11 & 21\end{array}\right]$ \\
\hline 4 & None \\
\hline 5 & None \\
\hline 6 & [151 6034 48]; [139 5829 43] \\
\hline 7 & [194 653847 32]; [182 653443 28] \\
\hline 8 & [108 4716 36]; ; 106 4615 36] \\
\hline 9 & None \\
\hline 10 & None \\
\hline
\end{tabular}

Figure 4 Comparisons of non- punctuality rate under two plans

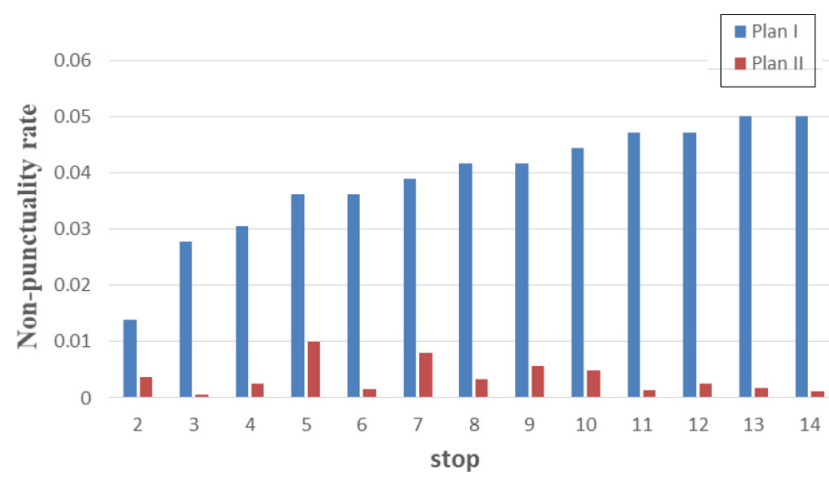

downstream stops and the non-punctuality rate would increase with time. Under Plan I, the average non-punctuality rate at the terminal stop is the largest which is up to 0.05 . In addition, the average non-punctuality rate between Stop 2 and Stop 3 increases significantly because there are two signalized intersections between them and results in unstable bus operations.

Under Plan II, the average non-punctuality rate at stop 3 is the minimum, while at Stop 5 is the maximum. There is because there are more road sections and intersections between Stop 2 and Stop 3, the speed guidance and signal adjustment can be applied to buses before arriving at Stop 3. However, there is only one road section between Stop 4 and Stop 5, only speed guidance can be applied to the buses before arriving at Stop 5.

\subsubsection{The variation of saturation degree in Plan II}

To reduce the non-punctuality rate, some signalized intersections would adjust their signal timing plans and result in the change of saturation degrees. Average variations of saturation degree under Plan II are plotted in Figure 5, where the maximum variation of saturation degree is 0.019
Figure 5 Saturation degree deviations of the five intersections

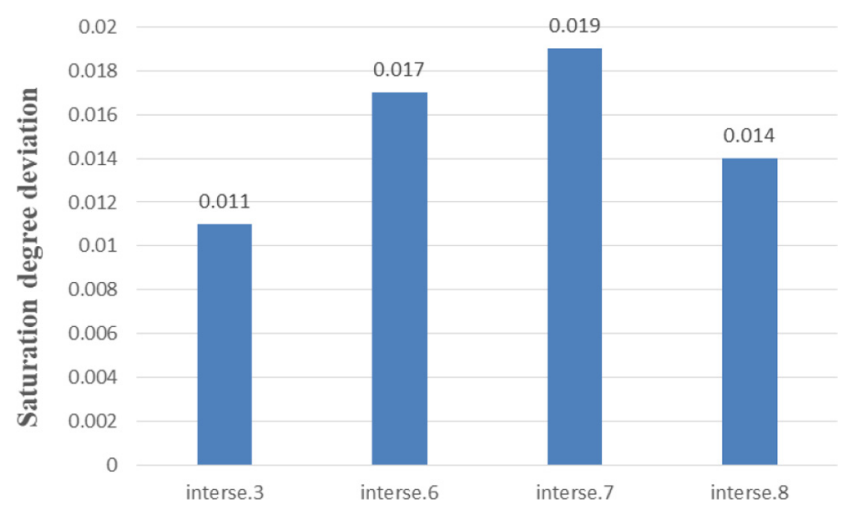

(at intersection 7). Compared with Plan I, the saturation degree of intersections under Plan II have slight variety. This result reveals that the adjustment of signal timing plan has an inconsiderable effect on the saturation degree of intersection.

To some extent, it is not enough to only control the bus operating speeds on road sections. Signal timing plan of the intersections also needs to be adjusted. The control center begins to optimize the signal timing plan when a bus enters the upstream road section, so it can adjust the signal control plans by multiple cycles in advance until the bus clear the intersection. In each cycle the timing parameters are only slightly adjusted. In such condition, the effect of dynamic bus control on saturation degree deviation is reduced. To dynamically control a bus, the adjusted timing plans of each intersection under Plan II are shown in Table 4 . From the table we can find that signal timing plans of intersections 3, 6, 7 and 8 are adjusted and that of other intersections are unchanged.

\subsubsection{Optimal objective values}

A bus running from one stop to its adjacent downstream stop is regarded as a stage. The speeds on road sections and signal timing plans at intersections would affect the objective value of each stage. Figure 6 compares the objective value of each stage between Plan I and Plan II. Although the objective value of Stage 6 is the maximum among all stages under Plan II, it is still smaller than the maximum value of each stage under Plan I. In addition, compared with Plan I, the total objective value under Plan II can be reduced by $78.4 \%$, from 0.51 to 0.11 .

Table 5 displays the contributions of different stages to the total objective value. In Plan I, the objective value of Stage 13 is 0.05 , which accounts for $9.8 \%$ of the total objective value, 0.51 . On the contrary, the proportion objective value in Stage 1 is relatively small. This is because in Plan I the buses are not controlled; thus, the non-punctuality rate increases with the bus travel distance. However, in Plan II, the objective value of Stage 13 only accounts for $1.1 \%$ of the total objective value. 
Figure 6 Comparisons in objective values between Plan I and Plan II

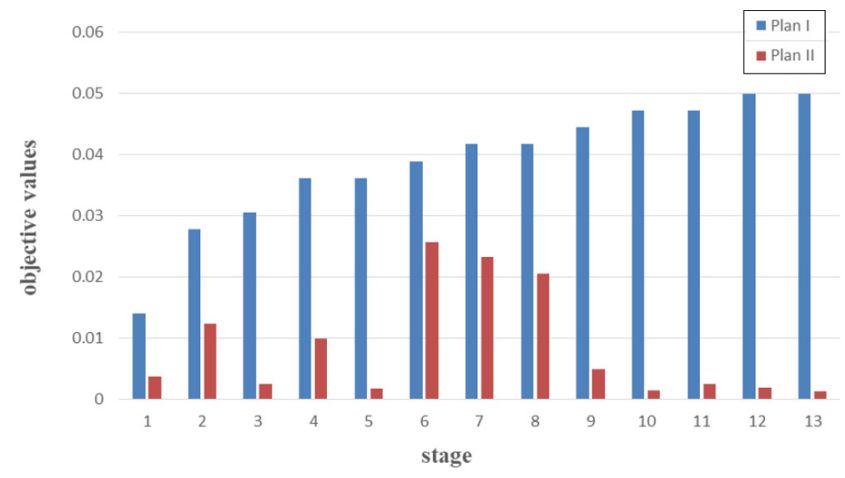

Table 5 Contribution percentages of each stage to the total objective value under each plan

\begin{tabular}{lccccccc}
\hline Stage & 1 & 2 & 3 & 4 & 5 & 6 & 7 \\
Plan I (\%) & 2.7 & 5.5 & 6.0 & 7.1 & 7.1 & 7.6 & 8.1 \\
Plan II (\%) & 3.4 & 11.2 & 2.2 & 9.0 & 1.5 & 23.2 & 21.1 \\
Stage & 8 & 9 & 10 & 11 & 12 & 13 & \\
Plan I (\%) & 8.1 & 8.7 & 9.2 & 9.2 & 9.8 & 9.8 & \\
Plan II (\%) & 18.6 & 4.5 & 1.2 & 2.3 & 1.6 & 1.1 & \\
\hline
\end{tabular}

\subsection{Bus operating speeds on different road sections}

In Plan I, the speeds on road sections are fixed, namely, $6.1 \mathrm{~m} / \mathrm{s}$. However, in Plan II, as shown in Table 6, we optimize the speed of each road section between $2.8 \mathrm{~m} / \mathrm{s}$ and $8.3 \mathrm{~m} / \mathrm{s}$ according to the length of the road section, the signal timing plan of the downstream intersection and the punctuality rate at the downstream stop. It proves that punctuality rate will increase with the number of road sections.

\subsubsection{Influence of weighting factor on the objective value}

Different values of $\omega$ would cause different total objective values under Plan II. As shown in Figure 7, the red histogram represents the total objective function value of Plan I, while the next 5 blue histograms represent the total objective function values corresponding to different $\omega$ in Plan II. Obviously, $\omega$ is set as 0.2 in the proposed strategy. Larger value of $\omega$ means the traffic regulator pays more emphasis on private traffics. If the regulator values punctuality more and hopes to give the public transit more priorities, $\omega$ should be set as a small value.

We can also find that when $\omega$ is greater than 0.5 , the total objective value does not increase as $\omega$ increases. This is because when $\omega$ is larger we should consider more about the benefits of private cases rather than buses. In such condition, only speed guidance on road sections can be applied and signal timing plan adjustment cannot be implemented. Hence, the saturation degree deviations of intersections are equal to 0 .

\section{Discussions}

In this section, we will analyze the influence of road geometry, intersection traffic flow on the optimization results and the quality of solutions.

\subsection{Impacts of road geometry on the optimization results}

The control model is carried out in the presence of a dedicated bus lane in this paper, so when the bus decelerates or accelerates on the road segment, it will not affect the operation of private traffics. However, if we consider that the research is performed without a dedicated bus lane, this control strategy will inevitably have a dynamic impact on the speed and delay of private vehicles. For example, if there is only one lane on the road section, the private vehicle will not be able to surpass the bus and will only run behind it; if there are multiple lanes on the road section, the private vehicle may overtake the bus. Since that the model becomes very intricate by describing this influence, the effect is ignored in the process of modeling. The model will take this impact into consideration in future studies. This is also one of the limitations of this study.

When the geometry of the intersection is different, buses have different effects on the overall traffic flow at intersections. Obviously, the more the number of approaches and the more the traffic flow, the greater the number of private traffics affected. Conversely, the fewer the number of private traffics affected. To simplify the model, we consider the effect of bus control scheme on saturation degree deviation as an evaluation index, which is closely related to the average delay of vehicles at the intersection and the average queue length. If the regulator wants to research the impact of bus control schemes on the traffic flow of all approaches at intersections, it is recommended to consider the total delay of vehicles as the evaluation index in equation (26).

Figure 7 Total objective values under different values of $\omega$

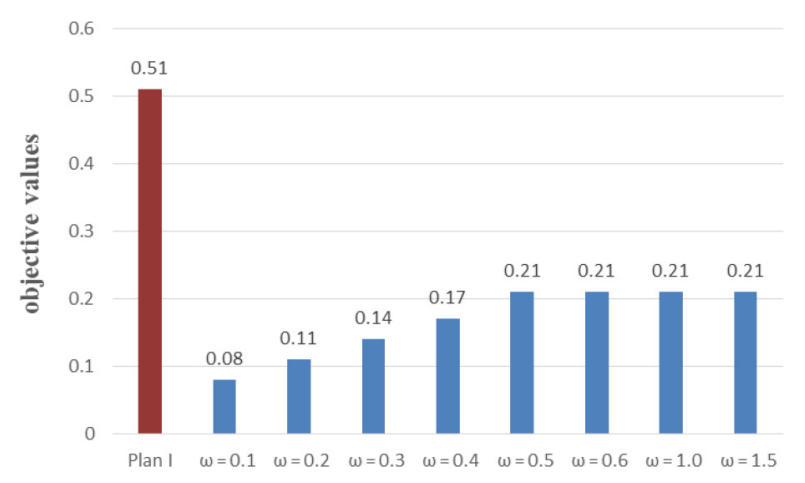

Table 6 Bus operating speeds on different road sections under plan II

\begin{tabular}{lcccccccccccc}
\hline Road section no. & 1 & 2 & 3 & 4 & 5 & 6 & 7 & 8 & 9 & 10 & 11 & 12 \\
Speed(m/s) & 5 & 7.2 & 5 & 8.3 & 8.3 & 7.2 & 5 & 6.1 & 3.9 & 5 & 2.8 & 5 \\
Road section no. & 13 & 14 & 15 & 16 & 17 & 18 & 19 & 20 & 21 & 22 & 23 & 7.1 \\
Speed(m/s) & 8.3 & 3.9 & 3.9 & 8.3 & 6.1 & 8.3 & 6.1 & 8.3 & 6.1 & 7.2 & 7.2 \\
\hline
\end{tabular}




\subsection{Impacts of intersection traffic demand on the optimization results}

There may be multiple states such as non-saturation and saturation at intersections in practical project, where we assume that each intersection is non-saturation. This study contains two optimization objectives which are saturation degree deviation and non-punctuality adherence. The first index mainly considers the proportion of intersection saturation change caused by bus control scheme. No matter what state the intersection is in, the same optimization result will be produced if the change of saturation degree is equal. For example, initial saturation degree in the intersection is equal to 0.80 under nonsaturation condition, while the saturation degree becomes 0.88 after control strategy implemented, and the saturation degree deviation is equal to 0.1 . However, initial saturation degree equals 1.1 under saturation condition, while the saturation degree becomes 1.21 after control strategy implemented, and the result is the same as the non-saturation condition, which is that saturation degree deviation is also equal to 0.1 . Therefore, the initial saturation degree has no impact on the saturation degree deviation.

\subsection{Discussions on the quality of solutions}

We establish the optimization model by using dynamic programming which is a good way to express multistage decision making and apply backward recursion solution to solve this problem which takes a long time to get the exact solution. When there are many stations on the bus line and multiple intersections, and the upstream section of the intersection is relatively long, the complexity of the model will rise rapidly, which may lead to curse of dimensionality. In this condition, backward recursion solution will not be suitable for the real-time dynamic control method established and we employ dimensional reduction to improve the real-time performance of model, such as reducing the speed interval of buses, reducing the number of signal cycles that can be adjusted at the intersection, or reducing the length of green time that can be adjusted by the phase of each signal.

\section{Conclusions}

This paper presents a bus dynamic control model for a route under connected bus environment. The proposed model aims to minimize the irregularity of the bus headways and the deviation of saturation degree at intersections, which is capable of mitigating the bus bunching problem for both bus curbside stops and bus bay. We divide the bus lines into stop, road section and intersection, and our model is on the premise of this precise division. The following conclusions can be summarized:

- We optimize the bus speed and signal timing plan jointly to eliminate the random disruptions along the route in connected bus environment, while reduces the negative effect on other vehicles to the utmost extent.

- We find that the signal control is not required when the road section is long enough. Otherwise, signal control will bring side effect on other vehicular to varying degrees. However, in most cases the effect only considering speed guidance is limited because of the velocity constraints.
- Signal control is one of the most promising options to mitigate the instability in bus system. In our paper, the signal timing plan is optimized once the bus enters the upstream road section, adjusting the signal plans for multiple cycles in advance and reducing the side effect on other vehicles.

The improvement of scheduled stability given by the proposed control scheme is on the premise that the speed guidance and signal control are precise. However, there may exits errors rising from the vagaries of bus drivers in speed guidance, compromising the effectiveness of this control. Thus, we will take this random error into consideration and optimize our model further in the future.

In this study, only one low-frequency bus line is considered when developing the method. In practice, there are multiple bus lines and some of them are high-frequency. In the next step, a more complex circumstance will be considered to improve our method.

\section{References}

Bie, Y., Gong, X. and Liu, Z. (2015), "Time of day intervals partition for bus schedule using GPS data", Transportation Research Part C: Emerging Technologies, Vol. 60, pp. 443-456.

Chen, S. and Sun, D. (2019), "A multistate-based travel time schedule model for fixed transit route", Transportation Letters, Vol. 11 No. 1, pp. 33-42.

Chung, E.H., Mahmoodi Nesheli, M. and Shalaby, A. (2020), "Transit holding control model for real-time connection protection", Fournal of Transportation Engineering, Part A: Systems, Vol. 146 No. 4, p. 04020021.

Dai, Z., Liu, X.C., Chen, Z., Guo, R. and Ma, X. (2019), "A predictive headway-based bus-holding strategy with dynamic control point selection: a cooperative game theory approach", Transportation Research Part B: Methodological, Vol. 125, pp. 29-51.

Estrada, M., Mensión, J., Aymamí, J.M. and Torres, L. (2016), "Bus control strategies in corridors with signalized intersections", Transportation Research Part C: Emerging Technologies, Vol. 71, pp. 500-520.

Gao, Q., Zhang, S., Chen, G. and Du, Y. (2020), "Two-way cooperative priority control of bus transit with stop capacity constraint”, Sustainability, Vol. 12 No. 4, p. 1405.

Gkiotsalitis, K. and Cats, O. (2018), "Reliable frequency determination: incorporating information on service uncertainty when setting dispatching headways", Transportation Research Part C: Emerging Technologies, Vol. 88, pp. 187-207.

Kumar, B.A., Prasath, G.H. and Vanajakshi, L. (2019), "Dynamic bus scheduling based on real-time demand and travel time", International fournal of Civil Engineering, Vol. 17 No. 9, pp. 1481-1489.

Liang, S., Zhao, S., Lu, C. and Ma, M. (2016), “A selfadaptive method to equalize headways: numerical analysis and comparison", Transportation Research Part B: Methodological, Vol. 87, pp. 33-43.

Steiner, K. and Irnich, S. (2018), "Schedule-based integrated intercity bus line planning via branch-and-cut", Transportation Science, Vol. 52 No. 4, pp. 882-897. 
Wang, S. and Qu, X. (2015), "Rural bus route design problem: model development and case studies", KSCE fournal of Civil Engineering, Vol. 19 No. 6, pp. 1892-1896.

Zhang, S. and Lo, H.K. (2018), "Two-way-looking selfequalizing headway control for bus operations", Transportation Research Part B: Methodological, Vol. 110, pp. 280-301.

Zhang, H., Liang, S., Zhao, J., He, S. and Zhao, T. (2020), "Coordinated headway-based control method to improve public transit reliability considering control points layout", Fournal of Advanced Transportation, Vol. 2020.

\section{Further reading}

Altun, S.Z. and Furth, P.G. (2009), "Scheduling buses to take advantage of transit signal priority", Transportation Research Record: Fournal of the Transportation Research Board, Vol. 2111 No. 1, pp. 50-59.

Cats, O., Larijani, A.N., Koutsopoulos, H.N. and Burghout, W. (2011), "Impacts of holding control strategies on transit performance: bus simulation model analysis", Transportation Research Record: Fournal of the Transportation Research Board, Vol. 2216 No. 1, pp. 51-58.

Chandrasekar, P., Long Cheu, R. and Chin, H.C. (2002), "Simulation evaluation of route-based control of bus operations", Fournal of Transportation Engineering, Vol. 128 No. 6, pp. 519-527.

Chen, Q., Adida, E. and Lin, J. (2013), "Implementation of an iterative headway-based bus holding strategy with real-time information", Public Transport, Vol. 4 No. 3, pp. 165-186.

Daganzo, C.F. (2009), "A headway-based approach to eliminate bus bunching: systematic analysis and comparisons", Transportation Research Part B: Methodological, Vol. 43 No. 10, pp. 913-921.

Hamdouch, Y., Szeto, W.Y. and Jiang, Y. (2014), "A new schedule-based transit assignment model with travel strategies and supply uncertainties", Transportation Research Part B: Methodological, Vol. 67, pp. 35-67.

Huang, D., Gu, Y., Wang, S., Liu, Z. and Zhang, W. (2020), "A two-phase optimization model for the demandresponsive customized bus network design", Transportation Research Part C: Emerging Technologies, Vol. 111, pp. 1-21.

Liu, Y., Liu, Z. and Jia, R. (2019), "DeepPF: a deep learning based architecture for metro passenger flow prediction",
Transportation Research Part C: Emerging Technologies, Vol. 101, pp. 18-34.

Liu, Z., Yan, Y., Qu, X. and Zhang, Y. (2013), "Bus stopskipping scheme with random travel time", Transportation Research Part C: Emerging Technologies, Vol. 35, pp. 46-56.

Osuna, E.E. and Newell, G.F. (1972), "Control strategies for an idealized public transportation system", Transportation Science, Vol. 6 No. 1, pp. 52-72.

Sánchez-Martínez, G.E., Koutsopoulos, H.N. and Wilson, N.H. (2016), "Real-time holding control for high-frequency transit with dynamics", Transportation Research Part B: Methodological, Vol. 83, pp. 1-19.

Tong, C.O. and Wong, S.C. (1999), "A schedule-based timedependent trip assignment model for transit networks", Fournal of Advanced Transportation, Vol. 33 No. 3, pp. 371-388.

Wang, S. and Qu, X. (2017), "Station choice for Australian commuter rail lines: equilibrium and optimal fare design", European fournal of Operational Research, Vol. 258 No. 1, pp. 144-154.

Wang, S., Qu, X. and Yang, Y. (2015), "Estimation of the perceived value of transit time for containerized cargoes", Transportation Research Part A: Policy and Practice, Vol. 78, pp. 298-308.

Xuan, Y., Argote, J. and Daganzo, C.F. (2011), "Dynamic bus holding strategies for schedule reliability: optimal linear control and performance analysis", Transportation Research Part B: Methodological, Vol. 45 No. 10, pp. 1831-1845.

Yu, B. and Yang, Z. (2009), "A dynamic holding strategy in public transit systems with real-time information", Applied Intelligence, Vol. 31 No. 1, pp. 69-80.

Yan, Y., Liu, Z., Meng, Q. and Jiang, Y. (2013), "Robust optimization model of bus transit network design with stochastic travel time", fournal of Transportation Engineering, Vol. 139 No. 6, pp. 625-634.

Yu, H., Chen, D., Wu, Z., Ma, X. and Wang, Y. (2016), "Headway-based bus bunching prediction using transit smart card data", Transportation Research Part C: Emerging Technologies, Vol. 72, pp. 45-59.

Zhao, J., Dessouky, M. and Bukkapatnam, S. (2006), "Optimal slack time for schedule-based transit operations", Transportation Science, Vol. 40 No. 4, pp. 529-539.

\section{Corresponding author}

Le Zhang can be contacted at: le_zhang111@126.com 